\title{
NONUNIFORM EXPANSION OF THE YOUNGEST GALACTIC SUPERNOVA REMNANT G1.9+0.3
}

\author{
Kazimierz J. Borkowski ${ }^{1}$, Stephen P. Reynolds ${ }^{1}$, David A. Green ${ }^{2}$, Una Hwang $^{3}$, Robert Petre ${ }^{4}$, \\ Kalyani KRISHNAMURThY ${ }^{5}$, AND ReBECCA Willett ${ }^{6}$ \\ ${ }^{1}$ Department of Physics, North Carolina State University, Raleigh, NC 27695-8202, USA; kborkow@ unity.ncsu.edu \\ ${ }^{2}$ Cavendish Laboratory, 19 J.J. Thomson Avenue, Cambridge CB3 OHE, UK \\ ${ }^{3}$ Department of Astronomy, University of Maryland, College Park, MD 20742, USA \\ ${ }^{4}$ NASA/GSFC, Code 660, Greenbelt, MD 20771, USA \\ ${ }^{5}$ Department of Electrical and Computer Engineering, Duke University, Durham, NC 27708, USA \\ ${ }^{6}$ Department of Electrical and Computing Engineering, University of Wisconsin-Madison, Madison, WI 53706, USA \\ Received 2014 May 29; accepted 2014 June 30; published 2014 July 11
}

\begin{abstract}
We report measurements of the X-ray expansion of the youngest Galactic supernova remnant, G1.9+0.3, using Chandra observations in 2007, 2009, and 2011. The measured rates strongly deviate from uniform expansion, decreasing radially by about $60 \%$ along the X-ray bright SE-NW axis from $0.84 \% \pm 0.06 \% \mathrm{yr}^{-1}$ to $0.52 \% \pm$ $0.03 \% \mathrm{yr}^{-1}$. This corresponds to undecelerated ages of 120-190 yr, confirming the young age of G1.9+0.3 and implying a significant deceleration of the blast wave. The synchrotron-dominated X-ray emission brightens at a rate of $1.9 \% \pm 0.4 \% \mathrm{yr}^{-1}$. We identify bright outer and inner rims with the blast wave and reverse shock, respectively. Sharp density gradients in either the ejecta or ambient medium are required to produce the sudden deceleration of the reverse shock or the blast wave implied by the large spread in expansion ages. The blast wave could have been decelerated recently by an encounter with a modest density discontinuity in the ambient medium, such as may be found at a wind termination shock, requiring strong mass loss in the progenitor. Alternatively, the reverse shock might have encountered an order-of-magnitude density discontinuity within the ejecta, such as may be found in pulsating delayed-detonation Type Ia models. We demonstrate that the blast wave is much more decelerated than the reverse shock in these models for remnants at ages similar to G1.9+0.3. Similar effects may also be produced by dense shells possibly associated with high-velocity features in Type Ia spectra. Accounting for the asymmetry of G1.9+0.3 will require more realistic three-dimensional Type Ia models.
\end{abstract}

Key words: ISM: individual objects (G1.9+0.3) - ISM: supernova remnants - X-rays: ISM

\section{INTRODUCTION}

G1.9+0.3 is the remnant of the most recent supernova (SN) in our Galaxy (Reynolds et al. 2008, Paper I). Its expansion of about $1.6 \%$ between 2007 and 2009 measured with Chandra (Carlton et al. 2011, Paper V) gives an expansion (undecelerated) age of about $160 \mathrm{yr}$, but the estimated mean expansion index $m\left(R \propto t^{m}\right)$ of about $m=0.7$ gives an age of $\sim 100 \mathrm{yr}$. The X-ray spectrum is dominantly synchrotron with high absorption (Reynolds et al. 2009, Paper III); lines of Fe, Si, and $\mathrm{S}$ are found in small regions with spectroscopic velocities of about 14,000 $\mathrm{km} \mathrm{s}^{-1}$ (Borkowski et al. 2010, Paper IV), consistent with the proper motions for a distance of the order of $10 \mathrm{kpc}$ (Roy \& Pal 2014). For an assumed location near the Galactic Center $(d=8.5 \mathrm{kpc})$, the diameter is about $2 \mathrm{pc}$. G1.9+0.3 is quite asymmetric (Figure 1), and the thermal emission is also asymmetrically distributed (Borkowski et al. 2013b, Paper VI). The relative prominence of the $\mathrm{Fe}$ lines, high velocities, the absence of a pulsar-wind nebula, and the bilaterally symmetric synchrotron emission (as in SN 1006) all point toward a Type Ia origin. Only a very unusual core-collapse event could reproduce the observations, while a reasonable SN Ia model can reach the observed size and velocity for a mean external density of about $0.02 \mathrm{~cm}^{-3}$ (Paper V). Observing the rapid evolution of G1.9+0.3 in morphology and brightness can provide unprecedented information concerning the dynamics of SN ejecta and on particle acceleration.

\section{OBSERVATIONS}

Chandra observed G1.9+0.3 with the ACIS S3 chip over three epochs: (I) 2007 February and March, (II) 2009 July, and
(III) 2011 May and July (details can be found in Papers I and III-VI). All observations were done in Very Faint Mode, and reprocessed with CIAO version 4.6 and CALDB 4.5.9. The corresponding effective exposure times are $49.6 \mathrm{ks}, 237 \mathrm{ks}$, and $977 \mathrm{ks}$. Exposure-weighted time intervals between the deep Epoch III and shorter Epoch I and II observations are $4.274 \mathrm{yr}$ and $1.861 \mathrm{yr}$. Alignment of observations at different epochs is performed simultaneously with expansion measurements.

Images $512^{2}$ pixels in size were extracted from the merged event files by binning event positions to half the ACIS pixel size, so one image pixel is $0^{\prime} \cdot 246 \times 0^{\prime} \cdot 246$. We also extracted data cubes $512^{2} \times 64$ in size using the same spatial pixel size, while spectral channels from 84 to $595(1.2-8.7 \mathrm{keV}$ energy range) were binned by a factor of eight.

$\mathrm{X}$-ray spectra were extracted from individual rather than merged event files and then summed (response files were averaged). Spectral analysis was performed with XSPEC version 12.8.1 (Arnaud 1996), using $C$-statistics (Cash 1979). The background was modeled rather than subtracted. Spectra of G1.9+0.3 were modeled with an absorbed power law, using the solar abundances of Grevesse \& Sauval (1998) in the phabs absorption model. Thermal emission contributes negligibly to broadband fluxes.

\section{EXPANSION}

We first measured the overall expansion using the method described in Paper V. Briefly, we smoothed the 2011 Chandra data cube with the spectro-spatial method of Krishnamurthy et al. (2010), and summed the smoothed cubes in the spectral dimension to arrive at smoothed images in the $1.2-8 \mathrm{keV}$ energy range. (Softer X-rays are absorbed by the intervening 
Table 1

Expansion Rates and Fluxes

\begin{tabular}{|c|c|c|c|c|c|c|c|c|c|}
\hline Region & $\begin{array}{l}\text { Expansion Rate } \\
\qquad\left(\% \mathrm{yr}^{-1}\right)\end{array}$ & $\begin{array}{r}\dot{S}_{2007^{\mathrm{a}}} \\
(\%)\end{array}$ & $\dot{\mathrm{S}}_{2009^{\mathrm{a}}}$ & $\begin{array}{c}N_{H}{ }^{\mathrm{b}} \\
\left(10^{22} \mathrm{~cm}^{-2}\right)\end{array}$ & $\Gamma^{\mathrm{c}}$ & $F_{2007^{\mathrm{d}}}$ & $\begin{array}{c}F_{2009}{ }^{\mathrm{d}} \\
\mathrm{g}^{-13} \mathrm{erg} \mathrm{cm}^{-2} \mathrm{~s}\end{array}$ & $F_{2011}{ }^{\mathrm{d}}$ & $\begin{array}{c}\text { Flux Rate Increase } \\
\left(\% \mathrm{yr}^{-1}\right)\end{array}$ \\
\hline Total & $\begin{array}{c}0.589 \\
(0.573,0.605)\end{array}$ & $\begin{array}{c}-0.2 \\
(-0.7,0.2)\end{array}$ & $\begin{array}{c}0.4 \\
(-0.1,0.9)\end{array}$ & $\begin{array}{c}7.25 \\
(7.16,7.34)\end{array}$ & $\begin{array}{c}2.40 \\
(2.37,2.43)\end{array}$ & $\begin{array}{c}27.34 \\
(26.75,27.93)\end{array}$ & $\begin{array}{c}28.79 \\
(28.50,29.08)\end{array}$ & $\begin{array}{c}29.73 \\
(29.57,29.99)\end{array}$ & $\begin{array}{c}1.9 \\
(1.5,2.3)\end{array}$ \\
\hline Outer SE-NW pair & $\begin{array}{c}0.523 \\
(0.487,0.560)\end{array}$ & $\begin{array}{c}0.0 \\
(-1.5,1.7)\end{array}$ & $\begin{array}{c}1.9 \\
(0.2,3.6)\end{array}$ & $\begin{array}{c}7.90 \\
(7.60,8.21)\end{array}$ & $\begin{array}{c}2.13 \\
(2.04,2.23)\end{array}$ & $\begin{array}{c}2.72 \\
(2.61,2.83)\end{array}$ & $\begin{array}{c}2.87 \\
(2.81,2.92)\end{array}$ & $\begin{array}{c}2.89 \\
(2.86,2.91)\end{array}$ & $\begin{array}{c}0.9 \\
(-0.3,2.2)\end{array}$ \\
\hline Middle SE-NW pair & $\begin{array}{c}0.616 \\
(0.592,0.640)\end{array}$ & $\begin{array}{c}-0.4 \\
(-1.1,0.4)\end{array}$ & $\begin{array}{c}1.0 \\
(0.2,1.8)\end{array}$ & $\begin{array}{c}7.58 \\
(7.45,7.72)\end{array}$ & $\begin{array}{c}2.34 \\
(2.29,2.38)\end{array}$ & $\begin{array}{c}9.16 \\
(8.97,9.35)\end{array}$ & $\begin{array}{c}9.75 \\
(9.66,9.84)\end{array}$ & $\begin{array}{c}10.05 \\
(10.00,10.09)\end{array}$ & $\begin{array}{c}1.9 \\
(1.3,2.5)\end{array}$ \\
\hline Inner SE-NW pair & $\begin{array}{c}0.842 \\
(0.783,0.898)\end{array}$ & $\begin{array}{c}0.3 \\
(-0.9,1.6)\end{array}$ & $\begin{array}{c}-0.3 \\
(-1.6,1.0)\end{array}$ & $\begin{array}{c}7.02 \\
(6.82,7.23)\end{array}$ & $\begin{array}{c}2.38 \\
(2.31,2.45)\end{array}$ & $\begin{array}{c}3.56 \\
(3.45,3.70)\end{array}$ & $\begin{array}{c}3.66 \\
(3.60,3.71)\end{array}$ & $\begin{array}{c}3.91 \\
(3.88,3.94)\end{array}$ & $\begin{array}{c}2.8 \\
(1.8,3.8)\end{array}$ \\
\hline $\mathrm{N}-\mathrm{S}$ pair & $\begin{array}{c}0.576 \\
(0.544,0.609)\end{array}$ & $\begin{array}{c}-0.2 \\
(-1.0,0.7)\end{array}$ & $\begin{array}{c}-0.2 \\
(-1.1,0.6)\end{array}$ & $\begin{array}{c}7.09 \\
(6.96,7.23)\end{array}$ & $\begin{array}{c}2.51 \\
(2.46,2.56)\end{array}$ & $\begin{array}{c}8.23 \\
(8.05,8.41)\end{array}$ & $\begin{array}{c}8.65 \\
(8.56,8.74)\end{array}$ & $\begin{array}{c}9.00 \\
(8.95,9.05)\end{array}$ & $\begin{array}{c}2.1 \\
(1.4,2.8)\end{array}$ \\
\hline
\end{tabular}

Notes. Expansion rates and fluxes in odd rows, $90 \%$ confidence limits in even rows.

a Surface brightness change.

b Hydrogen column density.

c Power-law photon index.

d Absorbed flux in the 1-7 keV energy range.

interstellar medium, while the background dominates at the highest energies.) We used the smoothed image as a model for the surface brightness of G1.9+0.3 at Epoch III (Figure 1). This model image was background-subtracted and then fit to the unsmoothed 1.2-8 keV images from earlier epochs (i.e., shrunk to fit) using $C$-statistics. Seven point sources within G1.9+0.3 were masked out. There are four free parameters in this model: a physical scaling factor, a surface-brightness scaling factor, and expansion center coordinates. Independent fits to Epoch I and II images were consistent with the constant expansion. We then assumed the same expansion rate while fitting jointly to the Epoch I and II images, but allowed for independent surfacebrightness scaling factors and expansion center coordinates. The results of all of our measurements are listed in Table 1. The measured expansion rate is $0.589 \% \pm 0.016 \% \mathrm{yr}^{-1}$ (all errors are at $90 \%$ confidence), in reasonable agreement with our previous measurement of $0.642 \% \pm 0.049 \%$ (Paper V). Within the errors, the mean surface brightness does not vary with time, again in agreement with Paper V.

To search for deviations from uniform expansion, we systematically examined the motions of large, distinct spatial structures. The final regions chosen are overlaid atop X-ray and radio images in Figure 1. They include a pair on the $\mathrm{N}$ and $\mathrm{S}$ rim and three pairs of regions along the major SE-NW axis: an outer pair ("ears"), a middle pair containing the brightest rim emission, and an inner pair with distinct interior rims.

Measurements of the expansion for each region pair were performed in the same way as for the entire remnant. We assumed a common expansion center of coordinates for all of the pairs for Epochs I and II, but the measured expansion rates are insensitive to this assumption. Slightly different region positions and areas (but not shapes) were used for Epochs I and II, to approximately match the measured expansion rates. The common expansion centers of the (J2000) coordinates are R.A. $17^{\mathrm{h}} 48^{\mathrm{m}} 45^{\mathrm{s}} .639 \pm 0.004\left(17^{\mathrm{h}} 48^{\mathrm{m}} 45^{\mathrm{s}} .629 \pm 0.002\right)$, decl. $-27^{\circ} 10^{\prime} 06^{\prime \prime} .85 \pm 0^{\prime \prime} .06\left(-27^{\circ} 10^{\prime} 06^{\prime \prime} .96 \pm 0^{\prime}\right.$. 03$)$ for Epoch I (II). A small ( 0.17$)$ but significant difference between these centers suggests that the coordinates of the corresponding reference observations ID6708 and ID10112 are slightly misaligned. The magnitude of this shift is consistent with the Chandra external astrometric errors (mean error of 0.'16; Rots 2009).

The measured expansion rates strongly deviate from uniform expansion (Table 1). Expansion rates increase inward by about $60 \%$ along the bright SE-NW axis, ranging from $0.52 \% \pm 0.03 \% \mathrm{yr}^{-1}$ for the outer ears to $0.84 \% \pm 0.06 \% \mathrm{yr}^{-1}$ for the inner rims. The bright rims in the middle expand slightly faster $\left(0.616 \% \pm 0.024 \% \mathrm{yr}^{-1}\right)$ than the ears, but even this small difference is statistically highly significant. The brightnessweighted linear displacement is $0^{\prime \prime} .29 \mathrm{yr}^{-1}$ for all three rims. The $\mathrm{N}-\mathrm{S}$ expansion is intermediate $\left(0.58 \% \pm 0.04 \% \mathrm{yr}^{-1}\right)$ between the bright middle rims and the ears, with an average displacement of $0.23 \mathrm{yr}^{-1}$. We demonstrate this differential expansion for two representative profiles in the SE and NW based on the Epoch II observations (Figures 2 and 3). As can be seen most clearly in the close-up inserts, the expansion rate (green) that matches the bright middle rims is too slow (too little shrinkage) for the inner rims, while the faster expansion rate (red) which matches the inner rims is too fast for the middle and outer rims.

Contributions to the $C$-statistic along the profiles quantify the fit quality. Systematic deviations are present even for the best-fit models, perhaps due to spatial variation of the Chandra point-spread function, smaller-scale spatial variations in the expansion rate, or projection effects. Strong counting noise limits the accuracy of the expansion measurements. However, these profiles are merely illustrative; the results in Table 1 are derived from the expansion of the entire regions shown in Figure 1.

\section{FLUX INCREASE}

The spatially integrated spectra of G1.9+0.3 from each epoch were jointly fit, together with their background spectra, with an absorbed power law in the $1-9 \mathrm{keV}$ energy range. The powerlaw index and absorbing column density were assumed to be constant in time, but the fluxes at each epoch were left free. There is good agreement with previous measurements for Epoch I, but the newly determined Epoch II flux is $2.5 \%$ larger, which disagrees with the previous measurement also reported in Paper $\mathrm{V}$, presumably due to updates in the ACIS S3 calibration that were applied to all three data sets.

Spatially integrated fluxes are clearly increasing with time (Table 1). A likelihood ratio test reveals that the linear flux increase is consistent with the individual flux measurements. The measured flux increase is $1.9 \% \pm 0.4 \% \mathrm{yr}^{-1}$, in agreement with the previous, less accurate value of $1.7 \% \pm 1.0 \% \mathrm{yr}^{-1}$ (Paper V). 

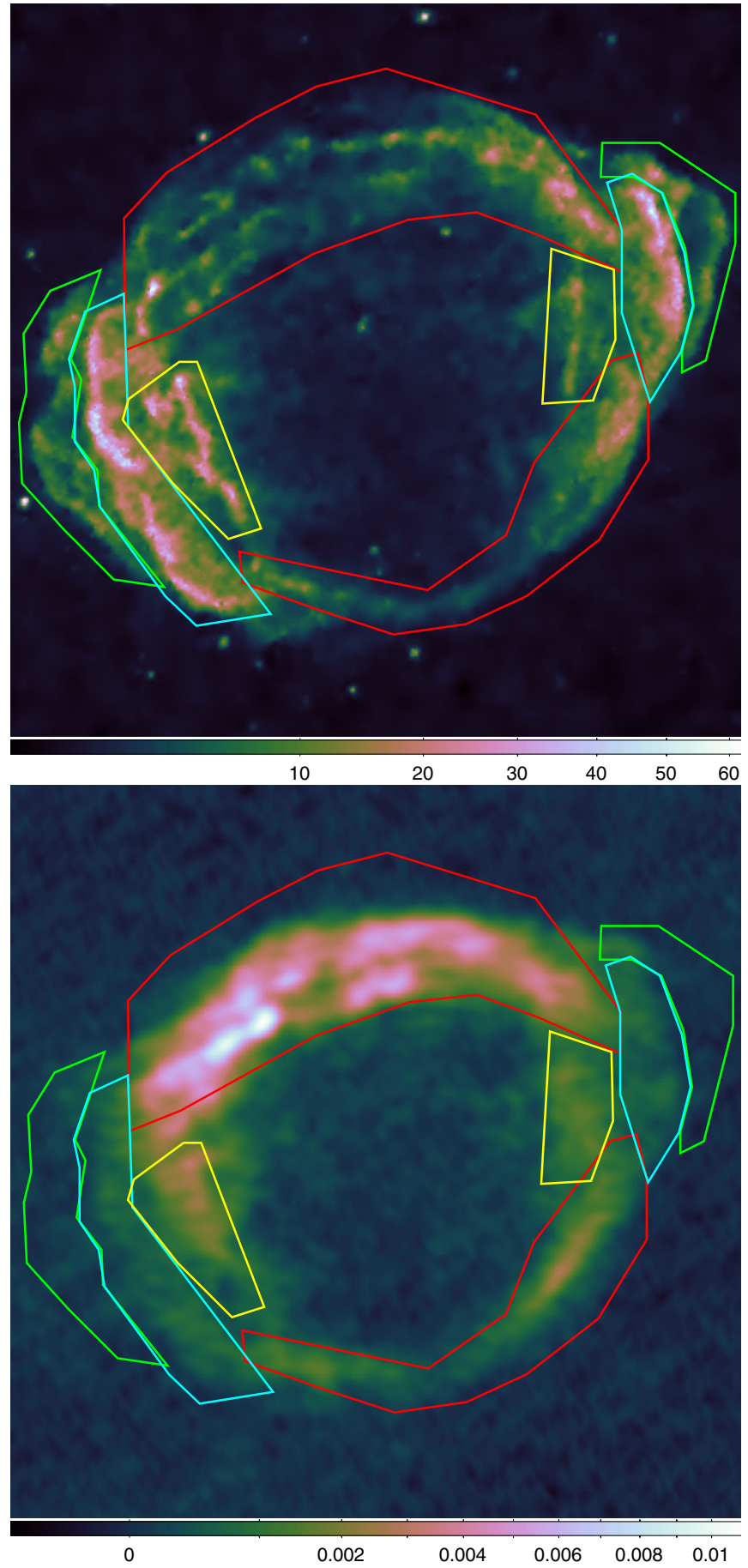

Figure 1. Top: 2011 Chandra image of G1.9+0.3. The scale is in counts per ACIS pixel in the $1.2-8 \mathrm{keV}$ energy range (image was smoothed with the multiscale partitioning method of Krishnamurthy et al. 2010). Bottom: $1.4 \mathrm{GHz}$ Very Large Array radio image from 2008 December. The scale is in Jy beam ${ }^{-1}$. The resolution is $2^{\prime \prime} .3 \times 1^{\prime \prime} .4$. $\mathrm{N}$ is up and $\mathrm{E}$ is to the left. Intensities are shown with the cubehelix color scheme of Green (2011). Pairs of regions chosen for expansion studies are overlaid: outer (green), middle (magenta), and inner (yellow) pairs along the SE-NW axis, and NS (red) pair. The image size is $123^{\prime \prime} \times 123^{\prime \prime}$.

We also measured the fluxes and rates of flux increase for the region pairs shown in Figure 1 in the same way as for the spatially integrated fluxes, except that the background contribution was scaled down (rather than fit again) by the region/total area ratios from the global fit. For each region pair, the measured rate of flux increase is consistent with the spatially integrated rate. There is an apparent trend in the brightening rate
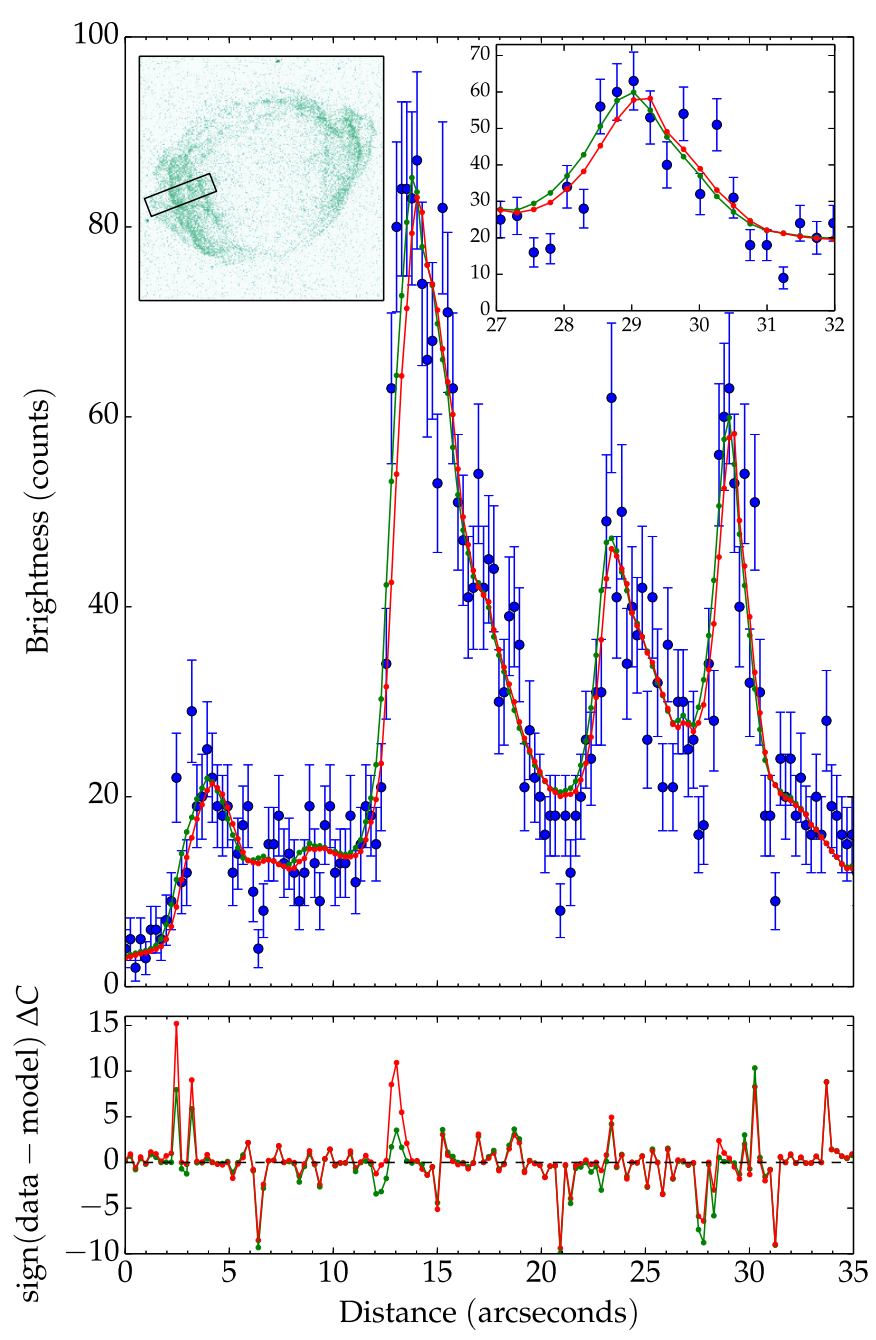

Figure 2. Top: SE profile from 2009 (blue) along the position shown on the 2009 image (left inset), together with model profiles corresponding to best-fit expansion rates of $0.62 \% \mathrm{yr}^{-1}$ (green) and $0.84 \% \mathrm{yr}^{-1}$ (red) for the middle and inner rims (see Figure 1 and Table 1). Right inset: zoomed view of the inner rim. Bottom: contribution to $C$-statistic. The horizontal scale is distance along the profile, measured from $\mathrm{E}$ to $\mathrm{W}$.

with radius, but uncertainties are large due to uncertainties in expanding the regions and to the dust scattering of emission from brighter regions (Paper III), as well as to large measurement errors. We conclude that evidence for spatial variations in the rate of flux increase is weak, although it should be the subject of future investigations.

\section{DISCUSSION}

The nonuniform expansion we observe for our three pairs of regions can be rephrased as large differences in the expansion age $\left(t_{\exp } \equiv \Delta t R / \Delta R\right)$, in the sense that $t_{\exp }$ is largest (greatest deceleration) for the slower-expanding outermost material (see Figure 1 and Table 1). The outermost ears, the bright rim emission in the middle, and the distinct interior rims all have measurably different expansion ages: $190 \mathrm{yr}, 160 \mathrm{yr}$, and $120 \mathrm{yr}$. We define the expansion indices $m \equiv d \ln r / d \ln t$ so that a feature at radius $r$ obeys $r \propto t^{m}$ (note that $d \ln r / d \ln t=$ $d \ln R / d \ln t$, where $R$ is the projection of the true radius $r$ onto the plane of the sky). Then, the true remnant age $t=m_{\mathrm{fw}} t_{\mathrm{exp}}$, where $m_{\mathrm{fw}}$ is the forward-shock expansion index. Since we only 


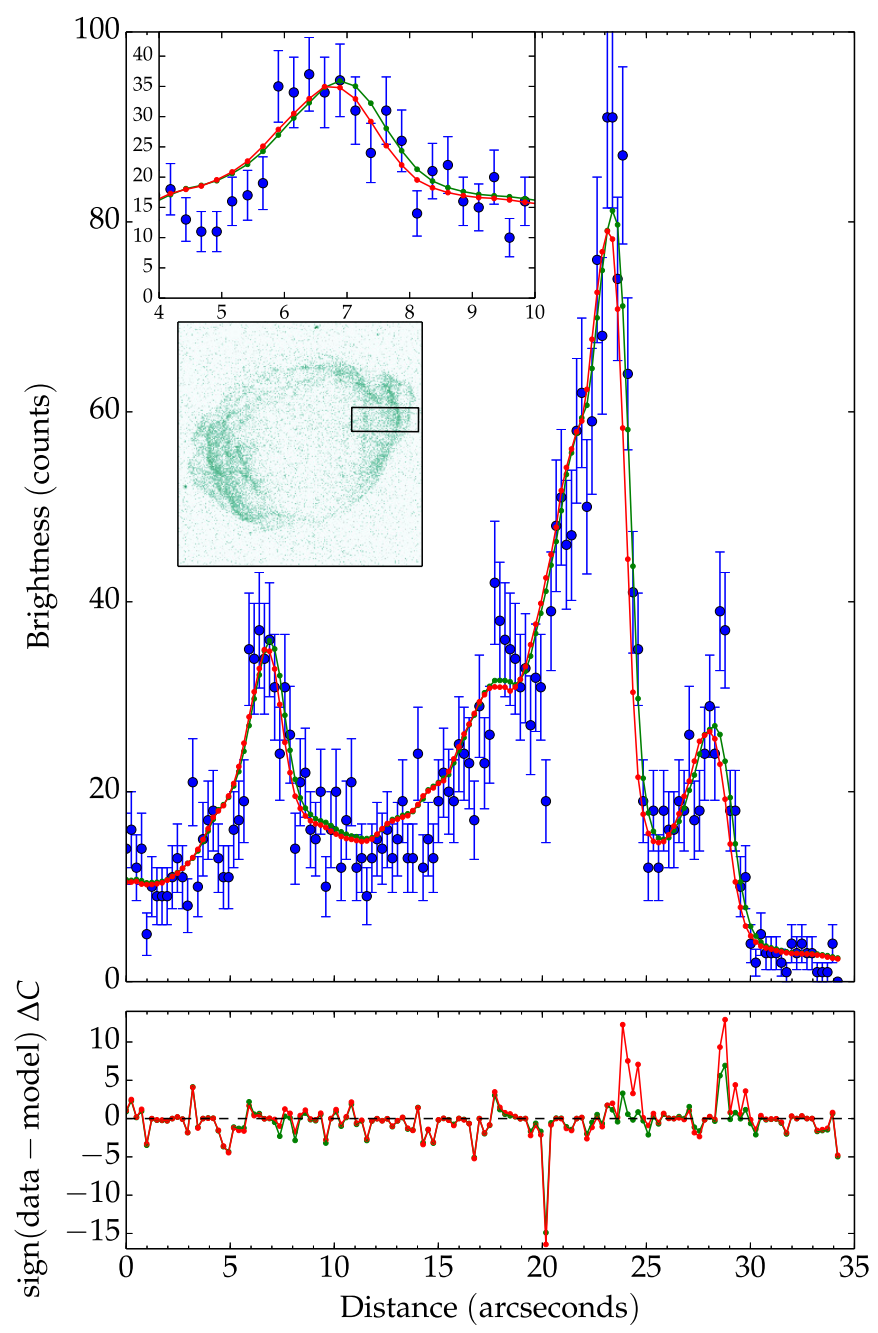

Figure 3. NW profile from 2009. See the caption of Figure 2 for explanation.

have an estimate of $100 \mathrm{yr}$ for the true remnant age (Paper V), we can only determine relative $m$ values.

The large spread in expansion ages between the inner rims and the ears implies a large deceleration of the forward shock, $m_{\mathrm{fw}} \lesssim 0.6$, significantly stronger than expected $\left(m_{\mathrm{fw}} \sim 0.7\right)$ in the models with smoothly varying density distributions for ejecta and ambient medium considered in Paper V. However, the more fundamental problem is that, contrary to observations, $m$ in these models typically varies only slightly (if at all) from the reverse shock to the blast wave, and it increases instead of decreasing with radius (Chevalier 1982; Dwarkadas \& Chevalier 1998, hereafter DC98). Either the ejecta or the ambient medium density distribution (or both) must be very different from the slowly varying density distributions considered so far. Various possibilities include substantial clumping or sudden density jumps within the ejecta or the ambient medium.

Fine-scale clumping within the ejecta is unlikely to explain the faster than expected expansion of the inner rims, however. Much like the outer rims, the inner rims consist of cohesive and continuous filaments in contrast to the much more clumpy north rim (Figure 1) where the ejecta emit most strongly in thermal X-rays (Paper VI). Another explanation involves a rapid deceleration of the blast wave following a recent encounter with a moderate density jump in the ambient medium. In order to account for the approximately $60 \%-70 \%$ slower expansion of the middle and outer rims relative to the inner rims (Table 1), a factor of 3-6 density jump is required (from Equations (4) and (6) in Borkowski et al. 1997). A density jump of this magnitude is consistent with a wind termination shock. If this were true, then the SN progenitor must have been losing mass in a strong and asymmetric stellar wind. We estimate the wind parameter ( $\equiv \dot{M} / v_{w}$, where $\dot{M}$ is the mass-loss rate and $v_{w}$ is the wind speed) at $4 \times 10^{-6} M_{\odot} \mathrm{yr}^{-1} / 1000 \mathrm{~km} \mathrm{~s}^{-1}$, using the analytic wind thin-shell solution for an exponential ejecta density distribution from Paper V with the remnant's radius of $2 \mathrm{pc}$ and undecelerated age of $120 \mathrm{yr}$, and assuming a standard thermonuclear explosion with a kinetic energy of $10^{51} \mathrm{erg}$ and an ejected mass equal to the Chandrasekhar mass. Such a strong wind favors a single-degenerate progenitor (Hachisu et al. 1996). The deceleration is $m=0.88$ in this model, suggesting that the explosion occurred sometime in the first decade of the 20th century. In this scenario, it is difficult to understand the origin of the strong north-south asymmetry seen at radio wavelengths (Figure 1) and in the spatial distribution of the thermal X-ray emission. As discussed in Paper VI, a strongly asymmetric Type Ia explosion provides the best explanation for this asymmetry.

Unlike the case for smooth ejecta density profiles (such as those resulting from delayed-detonation explosions), it is possible to obtain less deceleration inward $\left(m_{\mathrm{fw}}<m_{\mathrm{rev}}\right)$ in onedimensional (1D) numerical models for SNe Ia expanding into a uniform ambient medium for ejecta profiles with substantial structure (e.g., the PDDe model in Badenes et al. 2003). Prominent density structures in the outer ejecta layers are present in the deflagration models (e.g., the W7 model of Nomoto et al. 1984), sub-Chandrasekhar explosions (DC98; Badenes et al. 2003), and in pulsating delayed-detonation (PDD) models (e.g., Dessart et al. 2014, see their PDDEL1 model plotted in Figure 4). This density profile can be satisfactorily approximated by a power law $\rho \propto r^{-n}$ with $n=5.8$ above the transition velocity $v_{\text {tr }}=14,800 \mathrm{~km} \mathrm{~s}^{-1}$, and by the exponential model of DC98 at lower velocities. At $v_{\mathrm{tr}}$, there is a large (11.4) density jump.

In order to investigate how a density jump affects the speeds of the reverse and forward shocks, we performed 1D hydrodynamical simulations using the $\mathrm{VH}-1$ hydrocode (see Warren \& Blondin 2013 for a recent description of this code) for this composite power-law-exponential ejecta model. Here, we discuss the results for a preshock density $n_{0}$ of $0.2 \mathrm{~cm}^{-3}$, matching the preshock density found in a young Type Ia SNR 0509-67.5 in the Large Magellanic Cloud (Williams et al. 2011), but they can be scaled to any value of $n_{0}$ (DC98). Prior to the impact of the reverse shock with the density jump, the density profile can be described by the self-similar solutions of Chevalier (1982) with $m_{\mathrm{fw}}=m_{\mathrm{rev}}=(n-3) / n=0.48$ (see the density profile at $50 \mathrm{yr}$ in Figure 4). The reverse shock arrives at the density jump at $t=83\left(n_{0} / 0.2 \mathrm{~cm}^{-3}\right)^{-1 / 3} \mathrm{yr}$ with a velocity of $v_{s}=3 v_{\text {tr }} / n=0.52 v_{\text {tr }}$ (in the frame of reference moving with the ejecta), and then splits into transmitted and reflected shocks. The transmitted shock is the decelerated reverse shock (inward-facing). Its velocity is $v_{t}=(\beta / \delta)^{1 / 2} v_{s}$, where $\beta$ is the pressure enhancement that depends only on the density jump $\delta$ and varies between 1 and 6 (see Equation (6) in Borkowski et al. 1997). In the rest frame of the explosion, the deceleration of the transmitted reverse shock becomes $m_{\text {rev }}=1-3(\beta / \delta)^{1 / 2} / n$. With $\delta=11.4$, the overpressure is $\beta=2.76$ and $m_{\text {rev }}$ increases to 0.75 , in good agreement with hydrodynamical simulations (Figure 4). The reflected shock propagates first back into the shocked ejecta and then into the shocked ambient medium. At $100 \mathrm{yr}$, it has already passed through the low-density contact discontinuity that separates the shocked ejecta from the shocked 

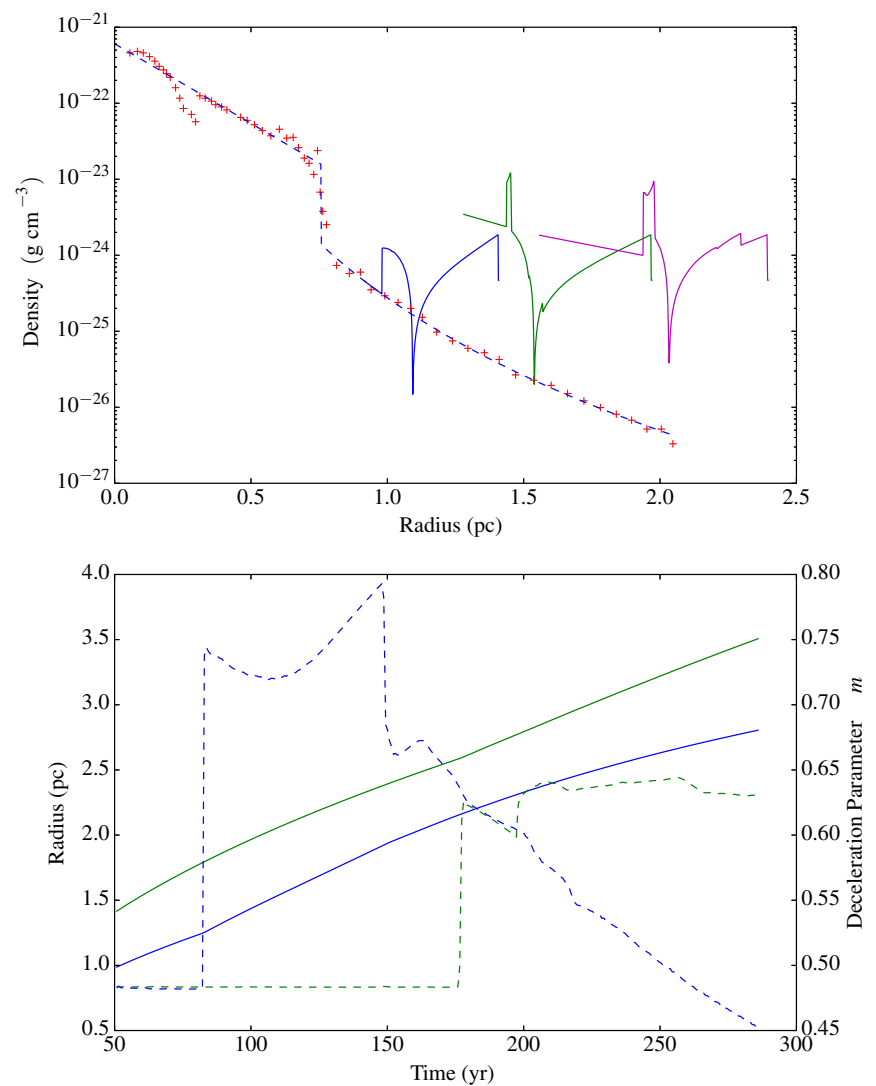

Figure 4. Top: density vs. radius in 1D hydrodynamical simulations with a composite power-law-exponential ejecta model and the uniform ambient medium with $n_{0}=0.2 \mathrm{~cm}^{-3}$ at $50 \mathrm{yr}$ (solid blue line), $100 \mathrm{yr}$ (green), and $150 \mathrm{yr}$ (magenta). Undecelerated (freely expanding) ejecta at $50 \mathrm{yr}$ are also shown: the composite model (dashed line) and the PDDEL1 model (Dessart et al. 2014) (+ signs). Bottom: radii of the reverse and forward shocks (in blue and green solid lines), and their deceleration parameters $m$ (dashed lines). The reverse shock expansion is faster than the blast wave expansion during a long $(\sim 100 \mathrm{yr})$ period of time.

ambient medium, and can be seen in Figure 4 as a small density discontinuity near the contact discontinuity. The reflected shock strengthens with time (see density profile at $150 \mathrm{yr}$ ), and eventually merges with the blast wave at $175 \mathrm{yr}$, resulting in an abrupt increase of $m_{\mathrm{fw}}$ from 0.48 to 0.62 . By this time, $m_{\mathrm{rev}}$ has already decreased to about the same value after a transient phase following the sudden deceleration of the reverse shock. This decrease continues in the subsequent evolution, while $m_{\mathrm{fw}}$ remains about constant, so that $m_{\mathrm{fw}}>m_{\mathrm{rev}}$.

During an extended period of time comparable to a young Type Ia remnant's age, PDD explosions show a more rapid expansion of the reverse shock than the forward shock (Figure 4). While this still might be a reasonable interpretation for G1.9+0.3, published PDD models fail to match its properties in detail. Assuming the ejecta structure of the composite model just described and identifying the blast wave with the bright middle rims at $r=2.04(d / 8.5 \mathrm{kpc}) \mathrm{pc}$ expanding at $0.62 \% \mathrm{yr}^{-1}$, the inferred preshock density $n_{0}$ and free expansion ejecta velocity at the reverse shock are $0.08(d / 8.5 \mathrm{kpc})^{-5.8}$ $\mathrm{cm}^{-3}$ and $18,000(d / 8.5 \mathrm{kpc}) \mathrm{km} \mathrm{s}^{-1}$. At $8.5 \mathrm{kpc}, n_{0}$ is several times higher than estimated in Paper $\mathrm{V}$, but the ejecta velocity is consistent with previous estimates. G1.9+0.3 is dynamically too young, however, for the reverse shock to have reached the density jump at $v_{\text {tr }}=14,800 \mathrm{~km} \mathrm{~s}^{-1}$. No published PDD model has $v_{\text {tr }}$ as high as $18,000 \mathrm{~km} \mathrm{~s}^{-1}$, with the highest $(16,000 \mathrm{~km}$ $\mathrm{s}^{-1}$ ) found in the PDDa model (Badenes et al. 2003). Among the models considered by Badenes et al. (2003), only the subChandrasekhar model SCH has a higher $v_{\text {tr }}$ at $18,500 \mathrm{~km} \mathrm{~s}^{-1}$. Furthermore, sub-Chandrasekhar explosions produce large (up to several $\times 10^{-3} M_{\odot}$ ) amounts of radioactive ${ }^{44} \mathrm{Ti}$ (e.g., Woosley $\&$ Kasen 2011), while the strength of the (possible) ${ }^{44} \mathrm{Sc}$ line implies that at most $\sim 10^{-5} M_{\odot}$ of ${ }^{44} \mathrm{Ti}$ was expelled by the SN that produced G1.9+0.3 (Borkowski et al. 2013a).

Only the outermost ( $\left.v \gtrsim 18,000(d / 8.5 \mathrm{kpc}) \mathrm{km} \mathrm{s}^{-1}\right)$ ejecta have been shocked so far in G1.9+0.3 (velocities might be as high as $v \gtrsim 21,000 \mathrm{~km} \mathrm{~s}^{-1}$ if $d \geqslant 10 \mathrm{kpc}$; Roy \& Pal 2014). Discrete absorption features with such extreme velocities are commonly seen in Type Ia SN spectra (e.g., Childress et al. 2014), perhaps indicating the presence of dense shells of material (Tanaka et al. 2006). Their origin is unknown, but possible explanations include a collision of ejecta with circumstellar medium or clumping within the ejecta. Fast dense shells with an order-of-magnitude density contrast relative to overlying tenuous ejecta are expected to decelerate the reverse shock just like the density jump in PDD models. This might be the explanation for the fast expansion of the reverse shock in G1.9+0.3.

The flux increase may be due to global effects such as an increase in magnetic-field strength, which would raise radio fluxes as well, and/or an increase in the maximum energy to which electrons are accelerated, which could raise X-ray fluxes even with constant radio. The radio flux of G1.9+0.3 is rising at $1 \%-2 \% \mathrm{yr}^{-1}$ (Green et al. 2008, Paper II; Murphy et al. 2008); our ongoing JVLA observations should reduce the uncertainty involved in determining whether this rate is consistent with the X-ray rate. A higher X-ray than radio rate of increase would require an increasing maximum energy. This would be a very interesting result. Spatial variations contain additional information. One might naively expect the greatest brightness increase at the location of greatest deceleration (thermalization of kinetic energy); our data suggest but do not compel this conclusion.

G1.9+0.3 offers us a unique opportunity to study the dynamics and spatial distribution of the outermost ejecta of a likely Type Ia $\mathrm{SN}$, and the process of particle acceleration in very fast collisionless shocks. Our current understanding of its dynamics and of the spatial variations in the flux rate increase is limited by the short-time baseline of the Epoch I-III observations and the poor signal to noise of the 2007 and 2009 Chandra data sets. Further Chandra observations will allow for significant advances in our understanding of G1.9+0.3.

This work was supported by NASA through Chandra General Observer Program grants SAO G01-12098A and B.

\section{REFERENCES}

Arnaud, K. A. 1996, in ASP Conf. Ser. 101, Astronomical Data Analysis and Systems V, ed. G. Jacoby \& J. Barnes (San Francisco, CA: ASP), 17

Badenes, C., Bravo, E., Borkowski, K. J., \& Domínguez, I. 2003, ApJ, 593, 358

Borkowski, K. J., Blondin, J. M., \& McCray, R. 1997, ApJ, 477, 281

Borkowski, K. J., Reynolds, S. P., Green, D. A., et al. 2010, ApJL, 724, L161 (Paper IV)

Borkowski, K. J., Reynolds, S. P., Green, D. A., et al. 2013a, BAAS, 13, 127.06

Borkowski, K. J., Reynolds, S. P., Hwang, U., et al. 2013b, ApJL, 771, L9 (Paper VI)

Carlton, A. K., Borkowski, K. J., Reynolds, S. P., et al. 2011, ApJL, 737, L22 (Paper V)

Cash, W. 1979, ApJ, 228, 939 
Chevalier, R. A. 1982, ApJ, 258, 790

Childress, M. J., Filippenko, A. V., Ganeshalingam, M., \& Schmidt, B. P. 2014, MNRAS, 437, 338

Dessart, L., Blondin, S., Hiller, D. J., \& Khokhlov, A. 2014, MNRAS, 441,532

Dwarkadas, V. V., \& Chevalier, R. A. 1998, ApJ, 497, 807 (DC98)

Green, D. A. 2011, BASI, 39, 289

Green, D. A., Reynolds, S. P., Borkowski, K. J., et al. 2008, MNRAS, 387, L54 (Paper II)

Grevesse, N., \& Sauval, A. J. 1998, SSRv, 85, 161

Hachisu, I., Kato, M., \& Nomoto, K. 1996, ApJL, 470, L97

Krishnamurthy, K., Raginsky, M., \& Willett, R. 2010, SIAM J. Imaging Sci., 3, 619

Murphy, T., Gaensler, B. M., \& Chatterjee, S. 2008, MNRAS, 389, L23
Nomoto, K., Thielemann, F.-K., \& Yokoi, K. 1984, ApJ, 286, 644

Reynolds, S. P., Borkowski, K. J., Green, D. A., et al. 2008, ApJL, 680, L41 (Paper I)

Reynolds, S. P., Borkowski, K. J., Green, D. A., et al. 2009, ApJL, 695, L149 (Paper III)

Rots, A. 2009, Determining the Astrometric Error in CSC Source Positions, http://cxc.harvard.edu/csc/memos/files/Rots_CSCAstrometricError.pdf

Roy, S., \& Pal, S. 2014, in IAU Symp. 296, Supernova Environmental Impacts, ed. A. Ray \& R. A. McCray (Cambridge: Cambridge Univ. Press), 197

Tanaka, M., Mazzali, P. A., Maeda, K., \& Nomoto, K. 2006, ApJ, 645, 470

Warren, D. C., \& Blondin, J. M. 2013, MNRAS, 429, 3099

Williams, B. J., Borkowski, K. J., Reynolds, S. P., et al. 2011, ApJ, 729,65

Woosley, S. E., \& Kasen, D. 2011, ApJ, 734, 38 